\title{
Hacia la Acreditación de la Universidad y las Escuelas de Postgrado
}

\author{
Máximo Ugarte Vega \\ Facultad Ciencias Administrativas de la UNMSM \\ Lima-Perú
}

\begin{abstract}
RESUMEN
Cuando hablamos de Globalización de la Economía y la sociedad de los conocımientos es preciso vincularlo con las universidades, porque la globalización exigente y competitiva también llegó a estos centros de estudios por lo tanto, las Universidades y las Escuelas de Postgrado tienen y deben estar a la altura de las exigencias de los acontecimientos, es decir, la globalización de los conocimientos.

Las Escuelas de Postgrado deben tener como misión apoyar y potencializar, con sistemas innovadores y altamente competitivos, el desarrollo y la especialización del recurso humano de nuestros departamentos y regiones; generando propuestas eficaces, viables, junto a la investigación básica y aplicada de acuerdo a las exigencias y necesidades de nuestro país, además de tener la autoevaluación y acreditación académica internacional.
\end{abstract}

Palabras clave: Estructura universitarias, presupuesto, mercado laboral, requisitos mínımos

\section{INTRODUCCION}

En ese sentido, trataremos de plantear tres aspectos resaltantes, de los muchos que pudieran ser, que a nuestro criterio se constituyen como los problemas en los que se encuentra la universidad peruana. El primero, la situación actual que vienen enfrentando la educación superior; el segundo, los principales factores de su entorno que influyen en la gestión de la universidad; y el tercer aspecto, sería comentar cuales son las perspectivas que tiene o debe tener la universidad y las escuelas de postgrado.

\section{SITUACIÓN ACTUAL Y PROBLEMAS $\mathrm{DE}$ LA UNIVERSIDAD.}

Los antecedentes de la Universidad peruana nos remontan a un centro de estudios tradicional, es decir colonial, oligárquica y de honorables, para luego institucionalizarse a inicios de 1919 bajo los intereses de una burguesía agraria - comercial de los terratenientes gamonales.

Con la Ley 13417 de 1960 se da inicio a la denominada «universidad moderna, profesional y reformada». Se reconocen las reivindicaciones reformistas como el gobierno universitario, la autonomía, gratuidad de la enseñanza, etc. En cambio, las leyes universitarias 17437 y 23733 llevaron a la frustración a estudiantes y profesores por ser insatisfactoria en los campos científico y técnico, especialmente esta última ley vigente, en la que se quería adecuar los patrones de dominación de una economía urbana. En la última década, la Universidad pierde su fuerza innovadora y movilizadora.

En su definición más amplia la Universidad viene a ser una institucion de enseñanza superior donde se cursan estudios en las facultades y se confieren los grados correspondientes. En ese contexto, 
se justifica que la generación de investigación y búsqueda de información se conviertan en conocimientos y éstos, a su vez, sean entregados a los alumnos. Asimismo, los objetivos fundamentales vienen a ser la formación académica, la investigación, la proyección hacia la sociedad, y hoy en día el objetivo viene a ser el de universidad empresa junto a los ideales y valores éticos que tienen que transmitirse $y$ difundirse en la Universidad.

La situación actual de la Universidad podemos sintetizarla en los siguientes aspectos:

a. Estructura universitaria. Antes de 1930 existían nueve universidades públicas y una privada; hoy tenemos en el Perú, aproximadamente 83 universidades; 33 son nacionales (públicas) y las demás son privadas. Del total de las universidades mencionadas, se calcula más de 573 programas de postgrado, de los cuales muchos estudian solo los fines de semana, por horas y el precio y la modalidad a convenir.

b. Población. En 1960 se matricularon 30,000 alumnos aproximadamente, y en la actualidad, según la Asamblea Nacional de Rectores (ANR) contamos con más de 500,000 estudiantes.

c. Presupuesto. Viene a ser uno de los problemas principales por el escaso apoyo del gobierno para incrementarlo, $y$ porque los pagos por derecho de matrícula que vienen a ser casi simbólicos. En términos generales el Presupuesto de la República del año 2003 ascendía a 1200 millones de nuevos soles que viene a ser el 3,4\% del PBI; y en el 2004 el $3,76 \%$ del PBI. De ese total, el $80 \%$ de estos presupuestos están destinados a pagar remuneraciones, siendo la partida más descuidada la de bienes y servicios que siguen deteriorándose desde hace dos décadas. En mayo del 2004, se realiza el denominado Pacto Social de los Compromisos Recíprocos por la Educación 2004-2006 (gobierno, partidos políticos, gremios empresariales y laborales, sociedad civil, etc., reunidos en el Foto del Acuerdo Nacional). El Pacto ratifica el compromiso de incrementar de $3,76 \%$ a $4,01 \%$ la participación de la educación en el PBI, sin embargo el presupuesto de educación del 2005 no incorpora dicho incremento; asimismo según fuentes del Ministerio de Educación indican que no progresa la inversión en educación desde el año 2000 , a pesar del crecimiento de la economía y del crecimiento de la presión tributaria del $12,1 \%$ al $13,4 \%$ del PBI.

En términos comparativos, somos uno de los países en Latinoamérica que menos gasta en educación por habitante: el Perú invierte 58 dólares al año; Bolivia 66; Colombia 97; Brasil 185 y Chile 238, según fuentes del CEPAL. Y, en el caso de las 30 universidades públicas reciben en conjunto la mitad de lo que gasta una universidad pública en Estados Unidos. En resumen no tenemos autonomía económica y seguimos atados a la burocracia estatal en el manejo del dinero, y las Escuelas de Postgrado en el Perú siguen autofinanciándose con ingresos propios y para algunas universidades las unidades de Postgrado son su principal fuente de ingresos o caja chica de gastos de sus autoridades.

d. La normativa jurídica. Se basa en la actual Ley Universitaria $N^{\circ} 23733$ de 1983, que regula las universidades públicas y 
privadas. En 1996 se promulga el Decreto Legislativo 882, mediante el que se aprueba la Ley de Promoción de la Inversión en la Educación. Hoy en día ambas normas resultan desfasadas porque no corresponden a los requerimientos y proyección de la universidad. La nueva Ley Universitaria (próxima a promulgarse), debe permitir en el ámbito económico, la ejecución de proyectos con total libertad.

e. Mercado laboral. La universidad, en general, no responde a las exigencias y necesidades de las organizaciones públicas y privadas en el país.

f. Número de egresados. Los egresados no corresponden al número de graduados. Por citar un ejemplo, el $90 \%$ de ingresantes a una Maestría o Doctorado, no llega a graduarse por diferentes aspectos, entre ellos, fundamentalmente, por la falta de certificación del idioma y la falta de metodología de investigación, en otras palabras superemos el síndrome del TMT (Todo Menos Tesis).

\section{FACTORES DEL ENTORNO QUE INFLUYEN EN LA UNIVERSIDAD.}

Todos somos conscientes de distintos factores que influyen en la Universidad, tanto en lo interno como en lo externo. Como factores internos podemos citar, entre otros, las deficiencias académicas, falta de docentes idóneos, deserción estudiantil, falta de diálogo y tolerancia, y resistencia al cambio, etc. En lo externo; por un lado, tenemos influencias como el gobierno, las empresas públicas y privadas, la sociedad, los profesores, estudiantes, trabajadores, $\mathrm{y}$ el entorno familiar. Y, por otro lado, está el ingreso de las universidades extranjeras al mercado nacional ofreciendo postgrados en forma tradicional y a distancia.

Las universidades tienen que afrontar la competitividad con una formación de profesionales que puedan responder a las exigencias actuales, en otras palabras tienen que avanzar de una visión regional a una internacional; la tecnología de la información como soporte para potenciar las interrelaciones entre los miembros de la comunidad académica (correo electrónico, Internet, educación a distancia, bibliotecas en línea, aulas virtuales, etc) es fundamental.

También influyen las nuevas exigencias como la competitividad y no la competencia (ser mejor que el otro), es decir la capacidad de gestión humana basada en la competitividad, donde las organizaciones como las universidades tienen retos a redefinir su visión, misión, estructura, estrategias y recursos, acciones orientadas a lograr mayores niveles de competitividad. Para el logro de estos propósitos se requiere de una estrategia humana orientada a ser generadora de valores a través de sus diferentes procesos como la selección, capacitación y desarrollo, compensacion, gestion del desempeño, diseño de cargos, reclutamiento, entre otros; asimismo, las perspectivas de aprender algo nuevo, junto a la psicología de la capacitación para el éxito, nos permitirán romper las barreras psicológicas de carácter decisivo para todas las formas de cambio. Todas estas redefiniciones exigirán terminar con el modelo tradicional, y en el caso de la Universidad de San Marcos con la cultura de la pobreza. 


\section{PERSPECTIVAS DE LAS ESCUELAS DE POSTGRADO}

En primer lugar, las Escuelas de Postgrado deben evaluar y acreditar su calidad mediante diferentes criterios y sistemas de acreditación, como el norteamericano y el de la Asamblea Nacional de Rectores, a través del Instituto de Investigación y Fomento de la Modernización y Acreditación Universitaria (ANR-INFOMACU,2001); esto es, un proceso que se convierte en el componente fundamental de cualquier esfuerzo que se haga para producir valor. En cambio, la evaluación es el acceso a través del cual se identifican los componentes esenciales de un objetivo de evaluación y se hace necesaria cuando se requere validar y hacer explícitos unos valores; examinar planes, acciones y logros; comprobar e interpretar los logros de un programa y/o mejorar un programa (Ejemplo, pre y post grado) o un curso de acción.

Asimismo, resaltamos, que todo proceso de evaluación de un programa presupone, entre otros, definir los propósitos de la evaluación; delimitar el objeto de evaluación; delimitar sus variables, elementos, indicadores y criterios, para establecer el nivel y calidad del programa de funcionamiento; así como recolectar, analizar e interpretar información pertinente; valorar la calidad del programa en su totalidad o en algunas de sus variables y elementos; analizar el logro del programa evaluado; identificar e incorporar los ajustes y cambios pertinentes; y establecer los procesos permanentes de seguimiento y control, en este caso de las Escuelas de Postgrado y que según opiniones de instituciones y otros expertos, se pueden medir por diferentes modalidades como la autoevaluación interna y externa. En el primero, la organización, ejecución y supervisión del programa de autoevaluación tiene que estar bajo responsabilidad $x$ personas que tengan el interés. compromiso con el programa. En segundo, participarían especialistas ajez: al programa de autoevaluacio= identificando los problemas, las dificultas= y sugiriendo propuestas más $=$ correcciones al programa.

En cambio, la acreditación impl. certificar públicamente los requerimient: mínimos de calidad que reúne un prograca o una institución académica sin la cual no podría mejorar el sistema universitario az país; en otras palabras, la acreditación tiex como propósito garantizar la calidad de la instituciones o programas de la universida y ayudarlos a mejorar sus niveles de caliax-

En esa orientación se pueden med. indicadores y criterios para establecer el nrsde calidad del programa en vigencia. í gestión académica y administrativa de .a Universidad, tales como: el prestigio de ?: Universidad y sus Postgrados, grador: académicos de sus profesores, selección admisión de alumnos, las distintas responsabilidades que asumen los graduados en las instituciones públicas y privadas de $\mathbf{l}_{2}$ sociedad, el currículo, el impacto social, lz investigación, número de publicaciones especializadas, horas de atención a los alumnos, infraestructura de sus aulas, bibliotecas, laboratorios, cafeterías, instalaciones deportivas, sistemas de apoyo para la búsqueda de empleo, entre otros. En resumen, se busca medir la eficiencia en la organización académica y de gestión del Postgrado.

Ahora, bien, para poder acreditar la calidad educativa, se tiene que plantear objetivos dentro de un plan estratégico, como formar profesionales de altísima 


\section{PERSPECTIVAS DE LAS ESCUELAS DE POSTGRADO}

En primer lugar, las Escuelas de Postgrado deben evaluar y acreditar su calidad mediante diferentes criterios y sistemas de acreditación, como el norteamericano y el de la Asamblea Nacional de Rectores, a través del Instituto de Investigación y Fomento de la Modernización y Acreditación Universitaria (ANR-INFOMACU,2001); esto es, un proceso que se convierte en el componente fundamental de cualquier esfuerzo que se haga para producir valor. En cambio, la evaluación es el acceso a través del cual se identifican los componentes esenciales de un objetivo de evaluación y se hace necesaria cuando se requiere validar y hacer explícitos unos valores; examinar planes, acciones y logros; comprobar e interpretar los logros de un programa y/o mejorar un programa (Ejemplo, pre y post grado) o un curso de acción.

Asimismo, resaltamos, que todo proceso de evaluación de un programa presupone, entre otros, definir los propósitos de la evaluación; delimitar el objeto de evaluación; delimitar sus variables, elementos, indicadores y criterios, para establecer el nivel y calidad del programa de funcionamiento; así como recolectar, analizar e interpretar información pertinente; valorar la calidad del programa en su totalidad o en algunas de sus variables y elementos; analizar el logro del programa evaluado; identificar e incorporar los ajustes y cambios pertinentes; y establecer los procesos permanentes de seguimiento y control, en este caso de las Escuelas de Postgrado y que según opiniones de instituciones y otros expertos, se pueden medir por diferentes modalidades como la autoevaluación interna y externa. En el primero, la organización, ejecución y supervisión del programa de autoevaluación tiene que estar bajo responsabilidad de personas que tengan el interés y compromiso con el programa. En el segundo, participarían especialistas ajenos al programa de autoevaluación, identificando los problemas, las dificultades y sugiriendo propuestas más no correcciones al programa.

En cambio, la acreditación implica certificar públicamente los requerimientos mínimos de calidad que reúne un programa o una institución académica sin la cual no se podría mejorar el sistema universitario del país; en otras palabras, la acreditación tiene como propósito garantizar la calidad de las instituciones o programas de la universidad y ayudarlos a mejorar sus niveles de calidad.

En esa orientación se pueden medir indicadores y criterios para establecer el nivel de calidad del programa en vigencia, la gestión académica y administrativa de la Universidad, tales como: el prestigio de la Universidad y sus Postgrados, grados académicos de sus profesores, selección y admisión de alumnos, las distintas responsabilidades que asumen los graduados en las instituciones públicas y privadas de la sociedad, el currículo, el impacto social, la investigación, número de publicaciones especializadas, horas de atención a los alumnos, infraestructura de sus aulas, bibliotecas, laboratorios, cafeterías, instalaciones deportivas, sistemas de apoyo para la búsqueda de empleo, entre otros. En resumen, se busca medir la eficiencia en la organización académica y de gestión del Postgrado.

Ahora, bien, para poder acreditar la calidad educativa, se tiene que plantear objetivos dentro de un plan estratégico, como formar profesionales de altísima 
calidad; la internacionalización con soportes de alianzas estratégicas con otras universidades; mejorar el posicionamiento en cuanto a los competidores; desarrollar programas de investigación con participación de docentes y estudiantes y/o en convenios de cooperación nacional e internacional; modernizar las currículas de las facultades y los syllabus de los profesores con un adecuado apoyo; modernizar la gestión con diferentes acciones a tomar en función de las políticas sobre administración: eficiencia y productividad; ética profesional, elemento fundamental para generar conciencia de sus responsabilidades en el desarrollo social, la producción y el fortalecimiento de una cultura organizacional; for mar líderes con una visión compartida y una cultura institucional de acuerdo a las exigencias de los cambios en la escena de la investigación, la rigurosidad, la tecnología y el desarrollo de competencias; incorporar también, en su gestión administrativa la tecnología de la información como parte de una gestión estratégica universitaria y vincular la universidad con la sociedad (no solos sus representantes) generando acciones institucionales que respondan a la necesidad de toda la sociedad.

Podemos decir, entonces, que las universidades deben ser dinámicas, creativas e innovadoras, junto a la tecnología de las comunicaciones permitan acercar la calidad de investigación, de enseñanza y de gestión.

Por lo expuesto, les invitamos a tener una visión compartida hacia un proyecto nacional de Universidades y Escuelas de Postgrado; actuar y comprometer nuestras voluntades para superar la situación actual de la Universidad y afrontar la competitividad, así como el fomento de la modernización a través de un sistema nacional de autoevaluación y acreditación con el propósito de garantizar la calidad de las instituciones universitarias y programas del postgrado en el Perú.

\section{REFERENCIAS}

1. Asamblea Nacional de Rectores. Rectorum Revista Oficial de la ANR; 2001.

- Universidad. Comisión de Coordinación Interuniversitaria; Dirección General de Desarrollo Académico; 2000.

2. Consejo Superior de Investigaciones de la UNMSM. Artículos y Notas Cinéticas publicados por autores Sanmarquinos en Revistas registradas en el ISI durante el 2004, Informe 07-CSI, 2005; en Taller sobre problemática y retos de la investigación en la UNMSM, 18 y 19 de Abril de 2005.

3. Informe sobre: la participación en la primera reunión de responsables de investigación de la Red de Macrouniversidades de América y el Caribe; en Mazatlán, Sinaloa, México, Informe 08-CSI, 2005; en Taller sobre problemática y retos de la investigación en la UNMSM, 18 y 19 de Abril de 2005.

- Boletín Nro. 28, del 28 al 13 de marzo de 2005.

- Boletín Nro. 44, enero-febrero, 2002.

4. Gonzalez Gonzalez, Jorge y otros. De la autoevaluación. Los paradigmas de la calidad educativa; México, 2004.

5. Meister, Jeanne. Universidades Empresariales. Editorial Mc. Gram Hill, Colombia; 2000. 